Vol. 49 (1994) [1-5]

\title{
A REMARK ON A THIRD-ORDER THREE-POINT BOUNDARY VALUE PROBLEM
}

\author{
Salvatore A. Marano
}

Let $f$ be a real function defined on $[0,1] \times R^{3}$ and let $\eta \in(0,1)$. Very recently, C.P. Gupta and V. Lakshimikantham, making use of the Leray-Schauder continuation theorem and Wirtinger-type inequalities, established an existence result for the problem

$$
\left\{\begin{array}{l}
x^{\prime \prime \prime}=f\left(t, x, x^{\prime}, x^{\prime \prime}\right) \\
x(0)=x(\eta)=x(1)=0
\end{array}\right.
$$

(Theorem 1 and Remark 4 of [Nonlinear Anal. 16 (1991), 949-957]).

The aim of the present paper is simply to point out how, by means of a completely different approach, it is possible to improve that result not only by requiring much more general conditions on $f$, but also by giving a precise pointwise estimate on $x^{\prime \prime \prime}$.

Let $f$ be a real function defined on $[0,1] \times \mathbb{R}^{3} ; \eta \in(0,1) ; k \in[1,+\infty) ; L^{k}([0,1])$ the space of all (equivalence classes of) measurable functions $\psi:[0,1] \rightarrow \mathbb{R}$ such that $\|\psi\|_{L^{k}([0,1])}=\left(\int_{0}^{1}|\psi(t)|^{k} d t\right)^{1 / k}<+\infty ; W^{3, k}([0,1])$ the space of all $u \in C^{2}([0,1])$ such that $u^{\prime \prime}$ is absolutely continuous in $[0,1]$ and $u^{\prime \prime \prime} \in L^{k}([0,1])$.

Consider the problem

$$
\left\{\begin{array}{l}
x^{\prime \prime \prime}=f\left(t, x, x^{\prime}, x^{\prime \prime}\right) \\
x(0)=x(\eta)=x(1)=0 .
\end{array}\right.
$$

A function $u:[0,1] \rightarrow \mathbb{R}$ is said to be a generalised solution of $(\mathrm{P})$ if $u \in W^{3, k}([0,1])$, $u(0)=u(\eta)=u(1)=0$ and, for almost every $t \in[0,1]$, one has $u^{\prime \prime \prime}(t)=$ $f\left(t, u(t), u^{\prime}(t), u^{\prime \prime}(t)\right)$.

Our interest in problem (P) originated reading [3]. In that paper the authors implicitly established the following existence theorem (see [3, Theorem 1 and Remark 4]).

Received 12 January 1993

Copyright Clearance Centre, Inc. Serial-fee code: 0004-9729/94 \$A2.00+0.00. 
ThEOREM A. Assume that:

(i $\left.i_{1}\right)$ the function $t \rightarrow f\left(t, z_{1}, z_{2}, z_{3}\right)$ is measurable for every $z_{1}, z_{2}, z_{3} \in \mathbb{R}$;

(i $\left.i_{2}\right)$ the function $\left(z_{1}, z_{2}, z_{3}\right) \rightarrow f\left(t, z_{1}, z_{2}, z_{3}\right)$ is continuous for almost every $t \in[0,1]$;

(is ) there exist $p, q, r, S \in L^{1}([0,1])$ such that, for almost every $t \in[0,1]$ and every $z_{1}, z_{2}, z_{3} \in \mathbb{R}$, one has

$$
\left|f\left(t, z_{1}, z_{2}, z_{3}\right)\right| \leqslant p(t)\left|z_{1}\right|+q(t)\left|z_{2}\right|+r(t)\left|z_{3}\right|+S(t) ;
$$

(i4) there exist $a, b, c \in \mathbb{R}$ and $\alpha \in L^{1}([0,1])$ such that, for almost every $t \in[0,1]$ and every $z_{1}, z_{2}, z_{3} \in \mathbb{R}$, one has

$$
f\left(t, z_{1}, z_{2}, z_{3}\right) z_{2} \geqslant a z_{2}^{2}+b\left|z_{1}\right|\left|z_{2}\right|+c\left|z_{2}\right|\left|z_{3}\right|+\alpha(t) z_{2} .
$$

Then, problem $(P)$ has at least one generalised solution $u \in W^{3,1}([0,1])$ provided

$$
\|r\|_{L^{1}([0,1])}<1
$$

and

$$
\begin{gathered}
\left(1-\|r\|_{L^{1}([0,1])}\right)\left(\frac{4}{\pi^{2}}|a|+\frac{4}{\pi^{3}}|b|+\frac{2}{\pi}|c|\right)+\frac{1}{\pi}\|p\|_{L^{1}([0,1])} \\
+\|q\|_{L^{1}([0,1])}+\|r\|_{L^{1}([0,1])}<1 .
\end{gathered}
$$

The method employed in [3], in proving Theorem A, consists of using the LeraySchauder continuation theorem and Wirtinger-type inequalities.

The aim of the present paper is simply to point out how, by means of a completely different approach based on the classical Schauder-Tychonoff fixed point theorem, it is possible to establish the following result, which improves Theorem A.

ThEOREM 1. Let the assumptions ( $\left.i_{1}\right),\left(i_{2}\right)$ and ( $\left.i_{3}\right)$ of Theorem $A$ be satisfied. Then problem $(P)$ has at least one generalised solution $u \in W^{3,1}([0,1])$ provided

$$
\frac{1}{8}\|p\|_{L^{1}([0,1])}+\frac{1}{2}\|q\|_{L^{1}([0,1])}+\|r\|_{L^{1}([0,1])}<1 .
$$

Moreover, for almost every $t \in[0,1]$, one has

$$
\left|u^{\prime \prime \prime}(t)\right| \leqslant \sup \left\{\left|f\left(t, z_{1}, z_{2}, z_{3}\right)\right|:\left|z_{1}\right| \leqslant \gamma / 8,\left|z_{2}\right| \leqslant \gamma / 2,\left|z_{3}\right| \leqslant \gamma\right\}
$$

where

$$
\gamma=\frac{\|S\|_{L^{1}([0,1])}}{1-\left(\frac{1}{8}\|p\|_{L^{1}([0,1])}+\frac{1}{2}\|q\|_{L^{1}([0,1])}+\|r\|_{L^{1}([0,1])}\right)}
$$

Before proving Theorem 1, we collect, in Proposition 1 below, the inequalities we shall use in the sequel. 
Proposition 1. Let $u \in W^{3,1}([0,1])$ be such that $u(0)=u(\eta)=u(1)=0$. Then one has:

(j1) $\max _{t \in[0,1]}|u(t)| \leqslant\left.\frac{1}{8} \max _{t \in[0,1]}\right|^{\prime \prime}(t) \mid ;$

(j2) $\max _{t \in[0,1]}\left|u^{\prime}(t)\right| \leqslant \frac{1}{2} \max _{t \in[0,1]}\left|u^{\prime \prime}(t)\right|$;

(js ) $\max _{t \in[0,1]}\left|u^{\prime \prime}(t)\right| \leqslant \int_{0}^{1}\left|u^{\prime \prime \prime}(t)\right| d t$.

Proof: The assertions $\left(j_{1}\right)$ and $\left(j_{2}\right)$ follow at once from Theorem 8.2 of [1]. The assertion $\left(j_{3}\right)$ derives from the fact that, owing to our assumptions, there exists $\xi \in[0,1]$ such that $u^{\prime \prime}(\xi)=0$.

We now give the following

Proof of Theorem 1: For every $z=\left(z_{1}, z_{2}, z_{3}\right) \in \mathbb{R}^{3}$ we put $\|z\|=$ $\max \left\{8\left|z_{1}\right|, 2\left|z_{2}\right|,\left|z_{3}\right|\right\}$. It is easy to check that the extended real function $M: t \mapsto$ sup $|f(t, z)|, t \in[0,1]$, where $\gamma$ is given by (1), is measurable. Moreover, owing to $\|z\| \leqslant \gamma$

(is), $M \in L^{1}([0,1])$.

Consider the set

$$
K=\left\{w \in L^{1}([0,1]):|w(t)| \leqslant M(t) \text { almost everywhere in }[0,1]\right\}
$$

Of course, $K$ is nonempty and convex. By the Dunford-Pettis theorem (see, for instance, [2], Theorem 1, p.101) it is also weakly compact. For every $w \in K$ and every $t \in[0,1]$, we put:

$$
\begin{gathered}
\alpha(w)=\frac{\eta}{2(1-\eta)} \int_{0}^{1}(1-\sigma)^{2} w(\sigma) d \sigma-\frac{1}{2 \eta(1-\eta)} \int_{0}^{\eta}(\eta-\sigma)^{2} w(\sigma) d \sigma \\
\beta(w)=\frac{1}{2 \eta(1-\eta)} \int_{0}^{\eta}(\eta-\sigma)^{2} w(\sigma) d \sigma-\frac{1}{2(1-\eta)} \int_{0}^{1}(1-\sigma)^{2} w(\sigma) d \sigma \\
\Phi(w)(t)=\frac{1}{2} \int_{0}^{t}(t-\sigma)^{2} w(\sigma) d \sigma+\alpha(w) t+\beta(w) t^{2} \\
\Psi(w)(t)=f\left(t, \Phi(w)(t), \Phi(w)^{\prime}(t), \Phi(w)^{\prime \prime}(t)\right)
\end{gathered}
$$

where, as usual, $\Phi(w)^{\prime}(t)=\frac{d \Phi(w)(t)}{d t}$ and $\Phi(w)^{\prime \prime}(t)=\frac{d^{2} \Phi(w)(t)}{d t^{2}}$. Let us prove that $\Psi(K) \subseteq K$. To this end, fix $w \in K$ and observe that, by (is) and (1), one has

$$
\begin{gathered}
\|w\|_{L^{1}([0,1])} \leqslant\|M\|_{L^{1}([0,1])}=\int_{0}^{1} \sup _{\substack{\|z\| \\
\| \gamma}}|f(t, z)| d t \\
\leq\left(\frac{1}{8}\|p\|_{L^{1}([0,1])}+\frac{1}{2}\|q\|_{L^{1}([0,1])}+\|r\|_{L^{1}([0,1])}\right) \gamma+\|S\|_{L^{1}([0,1])}=\gamma .
\end{gathered}
$$


Taking into account that $\Phi(w) \in W^{3,1}([0,1])$ and $\Phi(w)(0)=\Phi(w)(\eta)=\Phi(w)(1)=0$, from Proposition 1 it follows that

$$
\begin{gathered}
\max _{t \in[0,1]}|\Phi(w)(t)| \leqslant \frac{1}{8} \max _{t \in[0,1]}\left|\Phi(w)^{\prime \prime}(t)\right| \leqslant \frac{1}{8} \int_{0}^{1}|w(t)| d t \leqslant \frac{\gamma}{8} \\
\max _{t \in[0,1]}\left|\Phi(w)^{\prime}(t)\right| \leqslant \frac{1}{2} \max _{t \in[0,1]}\left|\Phi(w)^{\prime \prime}(t)\right| \leqslant \frac{1}{2} \int_{0}^{1}|w(t)| d t \leqslant \frac{\gamma}{2} \\
\max _{t \in[0,1]}\left|\Phi(w)^{\prime \prime}(t)\right| \leqslant \int_{0}^{1}|w(t)| d t \leqslant \gamma .
\end{gathered}
$$

Hence, $|\Psi(w)(t)| \leqslant \sup _{\|z\| \leqslant \gamma}|f(t, z)|=M(t)$ for almost every $t \in[0,1]$. This implies that $\Psi(w) \in K$.

Now, let us prove that the operator $\Psi$ is weakly continuous. Owing to the weak compactness of $K$, we need only verify that the graph $g r(\Psi)$ of $\Psi$ is weakly closed in $K \times K$. Taking into account Theorem 7, p.313, of [4], it suffices to show that $\operatorname{gr}(\Psi)$ is sequentially weakly closed. Let $w \in K$ and let $\left\{w_{n}\right\}$ be a sequence in $K$ weakly converging to $w$ in $L^{1}([0,1])$. From (2) it follows that, for every $t \in[0,1]$, $\lim _{n \rightarrow \infty} \Phi\left(w_{n}\right)(t)=\Phi(w)(t), \lim _{n \rightarrow \infty} \Phi\left(w_{n}\right)^{\prime}(t)=\Phi(w)^{\prime}(t)$ and $\lim _{n \rightarrow \infty} \Phi\left(w_{n}\right)^{\prime \prime}(t)=\Phi(w)^{\prime \prime}(t)$. Therefore, by $\left(i_{2}\right)$, the sequence $\left\{\Psi\left(w_{n}\right)\right\}$ converges almost everywhere in $[0,1]$ to $\Psi(w)$. Bearing in mind that for almost every $t \in[0,1]$ and every $n \in \mathbb{N}$ one has

$$
\left|\Psi\left(w_{n}\right)(t)\right| \leqslant M(t),
$$

by the Lebesgue dominated convergence theorem, we obtain that $\lim _{n \rightarrow \infty} \Psi\left(w_{n}\right)=\Psi(w)$ in $L^{1}([0,1])$. So, $\left\{\Psi\left(w_{n}\right)\right\}$ converges weakly to $\Psi(w)$.

At this point, we are allowed to apply the Schauder-Tychonoff fixed point theorem to $\Psi$. There is, therefore, $w \in K$ such that $w=\Psi(w)$. The function $u(t)=\Phi(w)(t)$, $t \in[0,1]$, satisfies our conclusion.

REMARK 1. We point out that Theorem 1 of [3] deals with the case $p, q, r \in L^{2}([0,1])$, $S \in L^{1}([0,1])$. It is easy to check that, in this case, the above-mentioned result and our Theorem 1 are mutually independent.

Taking into account that $\|\psi\|_{L^{1}([0,1])} \leqslant\|\psi\|_{L^{2}([0,1])}$ for every $\psi \in L^{2}([0,1])$, from Theorem 1, it is possible to derive the following

TheOREM 2. Let the assumptions $\left(i_{1}\right)$ and $\left(i_{2}\right)$ of Theorem $A$ be satisfied. Moreover, suppose that:

$\left(i_{3}^{\prime}\right)$ there exist $p, q, r, S \in L^{2}([0,1])$ such that, for almost every $t \in[0,1]$ and every $z_{1}, z_{2}, z_{3} \in \mathbf{R}$, one has

$$
\left|f\left(t, z_{1}, z_{2}, z_{3}\right)\right| \leqslant p(t)\left|z_{1}\right|+q(t)\left|z_{2}\right|+r(t)\left|z_{3}\right|+S(t) .
$$


Then, problem $(P)$ has at least one generalised solution $u \in W^{3,2}([0,1])$ provided

$$
\frac{1}{8}\|p\|_{L^{2}([0,1])}+\frac{1}{2}\|q\|_{L^{2}([0,1])}+\|r\|_{L^{2}([0,1])}<1 .
$$

Moreover, for almost every $t \in[0,1]$, one has

$$
\left|u^{\prime \prime \prime}(t)\right| \leqslant \sup \left\{\left|f\left(t, z_{1}, z_{2}, z_{3}\right)\right|:\left|z_{1}\right| \leqslant \gamma^{\prime} / 8,\left|z_{2}\right| \leqslant \gamma^{\prime} / 2,\left|z_{3}\right| \leqslant \gamma^{\prime}\right\},
$$

where

$$
\gamma^{\prime}=\frac{\|S\|_{L^{2}([0,1])}}{1-\left(\frac{1}{8}\|p\|_{L^{2}([0,1])}+\frac{1}{2}\|q\|_{L^{2}([0,1])}+\|r\|_{L^{2}([0,1])}\right)} .
$$

REMARK 2. The previous condition on $p, q$ and $r$ is not so restrictive as that requested in (2.17) or in Remark 4 of [3].

\section{REFERENCES}

[1] R.P. Agarwal, Boundary value problems for higher order differential equations (World Scientific, 1986).

[2] J. Diestel and J.J. Uhl Jr, Vector measures, Math. Surveys 15 (American Mathematical Society, Providence, R.I., 1977).

[3] C.P. Gupta and V. Lakshmikantham, 'Existence and uniqueness theorems for a third-order three-point boundary value problem', Nonlinear Anal. 16 (1991), 949-957.

[4] G. Köthe, Topological vector spaces - I (Springer-Verlag, Berlin, Heidelberg, New York, 1969).

Dipartimento di Matematica

Città Universitaria

Viale A. Doria 6

95125 Catania

Italy 